\title{
Random Changes of Accommodation Stimuli: An Automated Extension of the Flippers Accommodative Facility Test
}

Carles Otero, Mikel Aldaba, Silvia López, Fernando Díaz-Doutón, Fuensanta A. Vera-Díaz, and Jaume Pujol

\section{QUERY SHEET}

This page lists questions we have about your paper. The numbers displayed at left can be found in the text of the paper for reference. In addition, please review your paper as a whole for correctness.

Q1: $\quad$ Au: AQ: Please check edits in the title as per style sheet.

Q2: $\quad$ Au: Please provide missing affiliation (Department)

Q3: $\quad \mathrm{Au}$ : Please provide complete postal Address for corresponding Author.

Q4: $\quad$ Au: AQ: Keywords have been arranged alphabetically. Please check for correctness.

Q5: $\quad \mathrm{Au}$ : AQ: Please check the edit in the sentence 'Traditional measures of for correctness of intent.

Q6: $\quad$ Au: Please provide missing Publisher location for reference [5].

Q7: Au: Number of authors given in the reference entry [22] does not match with the number of authors to be present as per style.

\section{TABLE OF CONTENTS LISTING}

The table of contents for the journal will list your paper exactly as it appears below: 


\title{
Random Changes of Accommodation Stimuli: An Automated Extension of the Q1 DFlippers Accommodative Facility Test
}

\author{
Carles Otero ${ }^{\mathrm{a}}$, Mikel Aldabab ${ }^{\mathrm{b}}$, Silvia López ${ }^{\mathrm{a}}$, Fernando Díaz-Doutón ${ }^{\mathrm{b}}$, Fuensanta A. Vera-Díazc, and Jaume Pujol ${ }^{\mathrm{a}}$
}

Pavalor Research Center, Universitat Politècnica de Catalunya, Terrassa, Spain; ${ }^{b}$ Center for Sensors, Instruments and Systems Development, niversitat Politècnica de Catalunya, Terrassa, Spain; 'New England College of Optometry, Boston, MA, USA

\section{Introduction}

The ability of the eye to accurately and repeatedly change its accommodative state when changing focus between two focal planes during a certain period of time is clinically measured using the flippers accommodative facility test. ${ }^{1}$ This test is usually performed either at far distance (i.e., the fixation target is at 6-m distance) or at near distance (i.e., the fixation target is at 0.4-m distance), and the accommodative demand for each focal plane is lens-induced with an accommodation flipper. At near distance a pair of ophthalmic flipper lenses of $+2.00 \mathrm{D}$ and -2.00 $\mathrm{D}$, which stimulate, respectively, $+0.50 \mathrm{D}$ and $+4.50 \mathrm{D}$. At far distance, a lens of $-2.00 \mathrm{D}$ is used to stimulate an accommodative demand of $+2.17 \mathrm{D}$, and $+0.17 \mathrm{D}$ with no lens. The accommodative facility test is often performed in children ${ }^{2}$ and young adults when accommodation abnormalities are suspected. ${ }^{1}$ For children six to 12 years old, the expected (norm) finding is 6 cycles per minute (cpm) or more, when the test is performed monocularly in healthy subjects. ${ }^{2}$ For teenagers and young adults 13 to 30 years old, the expected finding is $11 \mathrm{cpm}$ or more. ${ }^{1}$ The accommodative facility results depend on the individual's amplitude of accommodation, e.g., prepresbyopic subjects from 30 to 42 years of age shown worse results than the previously cited normative values. ${ }^{3}$

Clinical accommodative facility tests are typically used as a measure of visual fatigue, ${ }^{4}$ which can be caused by accommodative (if used monocularly) and/or binocular vision (if used binocularly) dysfunctions. ${ }^{5}$ The tests are also used to evaluate the treatment effect of accommodation vision training. However, these tests measure accommodation responses under repeated and therefore predictable conditions for the patient, which is not what occurs in natural conditions. During normal daily activities, we are required to change focus within a vast range of focal planes, and in a random or pseudo-random fashion.

To our knowledge, accommodative facility tests have not been evaluated using more than two predictable accommodative demands, and for a specific viewing distance. Traditional measures of accommodative facility involved repeating the

\begin{abstract}
Purpose: To study the accommodative dynamics for predictable and unpredictable stimuli using manual and automated accommodative facility tests Materials and Methods: Seventeen young healthy subjects were tested monocularly in two consecutive
sessions, using five different conditions. Two conditions replicated the conventional monocular accommodative facility tests for far and near distances, performed with manually held flippers. The other three conditions were automated and conducted using an electro-optical system and open-field autorefractor.
Two of the three automated conditions replicated the predictable manual accommodative facility tests. The last automated condition was a hybrid approach using a novel method whereby far and near-

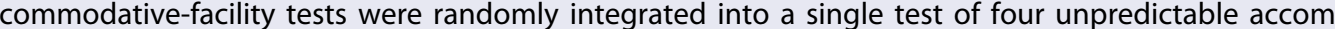
modative demands. were $( \pm 1, \pm 1)$ cycles per minute $(\mathrm{cpm})$ for the manual flipper accommodative facility conditions and $( \pm 3, \pm 4) \mathrm{cpm}$ for the automated conditions. The $95 \%$ limits of agreement between the manul and the unpredictable condition, the response time and accommodative response parameters were significantly $(p<0.05)$ larger for accommodation than disaccommodation responses for high accommodative demands only. The response times during the transitions $0.17 / 2.17 \mathrm{D}$ and $0.50 / 4.50 \mathrm{D}$ appeared to be indistinguishable between the hybrid unpredictable and the conventional predictable automated tests. results. Operator delays in flipping the lens may account for these differences. This novel test, using unpredictable stimuli, provides a more comprehensive examination of accommodative dynamics than conventional manual accommodative facility tests. Unexpectedly, the unpredictability of the stimulus did not to affect accommodation dynamics. Further studies are needed to evaluate the sensitivity of this
\end{abstract}


same accommodation demand change over a period of time; 70 herefore, these are predictable for the subject and do not Jonsider more than two accommodation planes. Emerging technologies such as computer-controlled electro-optical systems make it possible nowadays to change the spherical profile of an incoming wavefront by applying voltage to the system.

75 Electro-optical systems can use focus-tunable lenses, ${ }^{6}$ i.e., shape-changing lenses, based on a combination of optical fluids and a polymer membrane. Increasing the electric current pumps the liquid inside the lens and forces the membrane to deflect to a certain radius of curvature. This technology allows 80 inclusion of as many levels of accommodative demand as desired during the accommodative tests. It also allows randomization among accommodative demands so that they are not predictable. The features of this new technology have multiple useful applications because they allow: (1) automatization of

85 the test, (2) a more comprehensive examination of the patient's accommodation ability as multiple different accommodative demands may be measured, and (3) study of the potential effect of anticipation (due to stimulus' predictability). ${ }^{7-10}$ Nearly 50 years ago, ${ }^{8-10}$ it was suggested that if there is a repetitive

90 pattern driving the accommodation stimulus (as in accommodative facility tests), latency and response time for a certain accommodative change would be shortened. Latency is the time period between the start of the accommodative stimulus change and the start of the response of the subject, and 95 response time is the period between the start of the stimulus change and the moment the subject reports clarity. The effects of repetition in latency and response time would eventually lead to an overestimation of the accommodative facility measures.

In addition, a focus-tunable automated lens can be used to further understand the dynamics of accommodation when optically stimulated. This latter point is especially relevant because it has been shown that the steady-state accommodative response stimulated with lens-based systems is affected by

105 many factors such as the refractive error or the field of view when compared to free-space stimulation. ${ }^{11-13}$ Finally, a better understanding of the dynamics of accommodation under optical stimulation would provide insight into the visual discomfort that some individuals may experience in virtual rea-

110 lity systems. ${ }^{14}$ Visual fatigue has been associated with factors such as fast motion in depth, insufficient depth information, and unnatural types of blur. ${ }^{15}$

The purpose of this study is dual. First, we will compare the conventional manual flipper accommodative facility test 115 with an automated test performed in a computer-controlled electro-optical system; secondly, we will study accommodation dynamics with a new accommodative facility test that changes among various accommodative demands in a unpredictable manner.

\section{Materials and methods}

\section{Subjects}

The study was approved by the Ethics Committee of Hospital Mutua de Terrassa (Terrassa, Spain) and it followed the tenets of the Declaration of Helsinki. All subjects gave informed written consent. Criteria for inclusion were: (1) best-corrected visual acuity of $0.00 \operatorname{logMAR}(20 / 20$ Snellen equivalent) or better in each eye, (2) amplitude of accommodation above the average given by Hofstetter's formula for minimum accommodation $^{5}$ (Amplitude $=15-0.25^{\star} \mathrm{Age}$ ), (3) between 18 and 25 years of age, to ensure that the amplitude is not a confounding factor in the accommodative facility test, (4) spherical equivalent refractive error measured with subjective refraction between -6.50 and $+0.50 \mathrm{D}$ in each eye, (5) no strabismus, amblyopia, binocular, or accommodative anomalies, and (6) no history of any ocular disease, surgery, and/or pharmacological treatment that may have affected vision at the time of the study. Subjects with myopia wore their own disposable soft contact lenses for the study. All contact lenses prescriptions were within $\pm 0.25 \mathrm{D}$ of the subject's best correction in each meridian, determined by subjective refraction, as explained in the following sections.

\section{Instrumentation and methods}

The five different experimental conditions of this study that were randomly presented to each subject are summarized in Table 1. The first two conditions were manual clinical monocular accommodative facility tests, for far and near distances, respectively. The specific procedures for these two conditions were as follows: the examiner held accommodation/disaccommodation flipper glasses placed in front of the subject's eye at the eyeglasses plane, whereas the subject tried to clear the accommodative target described below. As soon as the subject reported clarity of the target, the examiner flipped the lenses to induce a change in the accommodative demand. Monocular accommodative facility was tested during $60 \mathrm{sec}-$ onds for each condition. The remaining three experimental conditions were conducted using an electro-optical system with and open-field autorefractor as described in Figure 1 and explained in detail below. For each of these three conditions, the subject was asked to report clarity of the accommodative target by pressing a key on a keyboard. At that point, the accommodative demand was automatically changed

Table 1. Summary of the experimental conditions. At far distance, the stimulus is placed at 6 meter $(0.17 \mathrm{D})$ and a lens of $-2.00 \mathrm{D}$ is used to stimulate an accommodative demand of $+2.17 \mathrm{D}$, and $+0.17 \mathrm{D}$ with no lens. At near distance, the stimulus is at $40 \mathrm{~cm}(2.50 \mathrm{D})$ and a pair of ophthalmic flipper lenses of +2.00 $\mathrm{D}$ and $-2.00 \mathrm{D}$ stimulate, respectively, +0.50 D and +4.50 D.

\begin{tabular}{|c|c|c|c|c|}
\hline Condition & Method & Distance & $\begin{array}{l}\text { Accommodative } \\
\text { transitions [D] }\end{array}$ & $\begin{array}{l}\text { Response } \\
\text { variables }\end{array}$ \\
\hline 1 & Manual flippers & Far & $0.17 / 2.17$ & Cycles/minute \\
\hline 2 & Manual flippers & Near & $0.50 / 4.50$ & Cycles/minute \\
\hline 3 & $\begin{array}{l}\text { Automated } \\
\text { (EOL system) }\end{array}$ & Far & $0.17 / 2.17$ & $\begin{array}{l}\text { Cycles/minute } \\
\text { Latency } \\
\text { Accommodative } \\
\text { response } \\
\text { Response time }\end{array}$ \\
\hline 4 & $\begin{array}{l}\text { Automated } \\
\text { (EOL system) }\end{array}$ & Near & $0.50 / 4.50$ & $\begin{array}{l}\text { Cycles/minute } \\
\text { Latency } \\
\text { Accommodative } \\
\text { response } \\
\text { Response time }\end{array}$ \\
\hline 5 & $\begin{array}{l}\text { Automated } \\
\text { (EOL system) }\end{array}$ & $\begin{array}{l}\text { Far \& Near } \\
\text { (hybrid } \\
\text { approach) }\end{array}$ & $0.17 / 0.50 / 2.17 / 4.50$ & $\begin{array}{l}\text { Latency } \\
\text { Accommodative } \\
\text { response } \\
\text { Response time }\end{array}$ \\
\hline
\end{tabular}



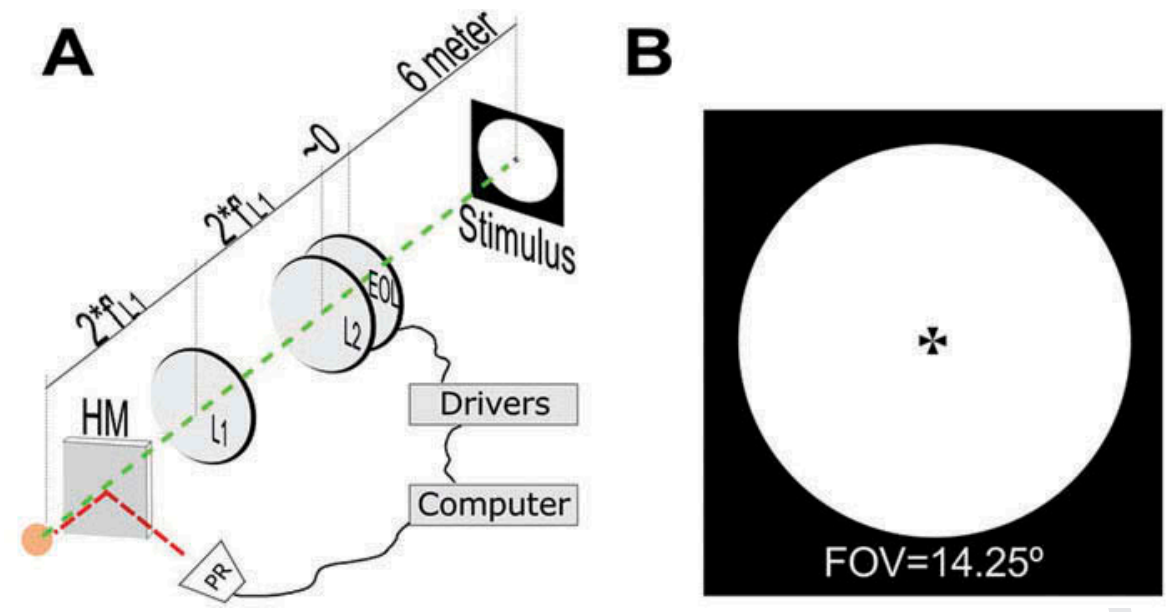

Figure 1. A: schematic view of the setup. B: accommodative stimulus used in the experiment. HM: Hot mirror. EOL: Electro-optical lens. PR: PowerRef II. f': focal length.

to the next accommodative level. Conditions 3 and 4 replicated the standard clinical far and near distance accommodative facility tests of condition 1 and 2 ; thus, the accommodative demand changed between 0.17 and 2.17 (far distance condition) or between 0.50 and 4.50 D (near distance condition). Finally, in condition 5, we integrated the far and near accommodative facility tests into one hybrid test that comprised four possible accommodative demands pseudorandomly chosen. Therefore, a total of six possible combinations of accommodation changes pairs were tested with four accommodative demands (i.e., $0.17 / 0.50,0.50 / 2.17,0.17 / 2.17$, $2.17 / 4.50,0.50 / 4.50,0.17 / 4.50)$. Each pair defined one possible transition that could be presented either for accommodation or disaccommodation. In order to ensure the same accommodative demand changes (or 'overall effort') in all subjects, the pseudo-random sequence forced eight times each possible transition (e.g., eight times the transition 0.17 to $2.17 \mathrm{D}$, eight times the transition 4.50 to $2.17 \mathrm{D}$, etc.). Condition 5 comprised of 48 transitions for accommodation and 48 transitions for disaccommodation that were randomly presented. Note that in order to compare the dynamics measured with the autorefractor among conditions 3,4, and 5, conditions 3 and 4 also ended once the subject had cleared the 48 transitions for accommodation (i.e., 0.18 to $2.17 \mathrm{D}$ for condition 3 and 0.50 to $4.50 \mathrm{D}$ for condition 4 ) and the 48 transitions for disaccommodation (i.e., 2.17 to $0.17 \mathrm{D}$ for condition 3 and 4.50 to $0.50 \mathrm{D}$ for condition 4 ).

A binocular open field autorefractor, PowerRef II

ing, a 50-mm square IR hot mirror was placed $40 \mathrm{~mm}$ from the subject's pupil plane. Subjects look at the accommodative stimulus through an optical system comprised by three lenses (Figure 1A). The first lens ( $\mathrm{L} 1$, diameter of $50 \mathrm{~mm}$, focal length of $100 \mathrm{~mm}$ ) was placed $200 \mathrm{~mm}$ from the subject's created $200 \mathrm{~mm}$ away from the lens, without magnification. The active module that performed the accommodation stimulation was placed in that plane and composed by an electrooptical lens ${ }^{6}$ (EOL, EL-16-40-TC, Optotune Switzerland AG, Switzerland) and a second lens (ophthalmic type) attached to it (L2, diameter of $25 \mathrm{~mm}$, power of $+3 \mathrm{D}$ ). The EOL had a spherical power range from -10 to $+10 \mathrm{D}$, with a reproducibility of $\pm 0.05 \mathrm{D}$ and a power settling time of $25 \mathrm{~ms}$ (according to manufacturer's specifications). The EOL power was controlled by a current driver, which was connected to a PC and controlled by means of a software application specifically developed for this study that synchronized the accommodative demand changes (for conditions 3, 4, and 5) with the PowerRef. In order to avoid possible thermal drifts on the EOL response, it was warmed up to $28^{\circ} \mathrm{C}$ before beginning the measurement sessions, and kept in that temperature throughout the procedures. Moreover, the EOL response at that temperature was calibrated before its integration on the system by means of a digital lensometer CL-300 (Topcon, Japan), including the calibration curve in the software application.

The target was placed $6 \mathrm{~m}$ away from the EOL. This design ensured both the linearity and the 1:1 relationship between the power applied by the EOL and the accommodation stimulated to the subject, as well a constant size of the stimulus when changing in the accommodative demand. The role of lens $\mathrm{L} 2$ was to shift $3 \mathrm{D}$ the working power range of the EOL in order to avoid its operation limits (far vision corresponds to an EOL power of $+7 \mathrm{D}$, instead of $+10 \mathrm{D})$, thus guaranteeing its best performance. The overall system can accurately measure an accommodative range up to $10.00 \mathrm{D}$, with a constant field of view of $14.25^{\circ}$ in diameter. The response time for each step change of accommodative demand was approximately $40 \mathrm{~ms}$ (response time of the electronics + settling time of the EOL).

The accommodative target for all five conditions was a $2^{\circ}$ high-contrast black Maltese cross on a white uniform background (Figure 1B). Even though this stimulus does not have peripheral depth cues, which could have improved the accommodative response, ${ }^{12,18}$ it is frequently used for accommodation studies due to its wide frequency spectrum of spatial pupil (twice $f_{L 1}$ ). In this way, a pupil conjugate plane was 
frequencies. According to Charman and Tucker, the accommodative response is most stable to stimuli of wide spatial bandwidth. ${ }^{19}$ In addition, this stimulus was chosen because it is easily reproducible and allows direct comparisons of our results with previous dynamic accommodation studies. ${ }^{20-22}$

\section{Examination protocol}

Monocular subjective refraction with endpoint criteria of maximum plus power that provides best visual acuity was per250 formed to determine best optical correction for each subject. Monocular amplitude of accommodation was evaluated by averaging the values of two push-up and two push-down trials to compensate for the bias of push-up to overestimate and push down to underestimate accommodation amplitude. ${ }^{23}$

The five experimental conditions previously described were measured twice in two separate sessions (test-retest, same day) that took approximately 30 minutes each, including breaks. Subjects were allowed to take breaks as needed, although there was no systematic method to provide rests during the mea-

260 surements. Randomization of configurations was rigorously applied to minimize potential learning or fatigue biases. The resting time between the two sessions was 15 minutes and none of the subjects were trained before starting the experiment. For all experimental conditions, the accommodation 265 response was measured monocularly with the contralateral eye occluded with an eye patch.

\section{Data analyses}

From each accommodation response, three parameters were obtained: Latency, response time, and accommodative response. 270 Latency was computed as described by Kasthurirangan et al. ${ }^{24}$ To automatically find the start of the response, an algorithm searched for three consecutive increasing data values, followed by four consecutive data values in which no two consecutive decreases occurred. When these criteria were met, the first data point in the sequence was selected as the start of the response. The inverse algorithm was used to determine the start of the disaccommodative response. Accommodation response time was computed as the time period (in seconds) between the start of the accommodative stimulus change and 280 the moment the subject reported clarity and pressed a key. The accommodative response at each accommodative demand (half-cycle) was computed as the difference in diopters between the median refraction of the last four samples and the median refraction of the first four samples. The last sample corresponded to the moment in which the subject reported clarity and the first sample corresponded to the start of the accommodative stimulus change. Notice that for the hybrid condition, only accommodation changes between 0.17 and $2.17 \mathrm{D}$ and between 0.50 and $4.50 \mathrm{D}$ were considered for the analyses.

Data were processed using Matlab R2015b (MathWorks, Inc., USA). Repeatability of the far and near accommodative facility for the manual conditions 1 and 2, and automated experimental conditions 3 and 4 were analyzed using withinsubject standard deviation and paired $t$-tests. Agreement between the manual flippers and the automated tests at both target distances were analyzed using the $95 \%$ limits of agreement and paired $t$-tests. In both of these analyses (repeatability and agreement), the response variable was the number of cycles per minute.

The differences between the hybrid accommodative facility test (condition 5) and the conditions 3 and 4 , all performed in the EOL system, were analyzed using a repeated measures ANOVA with three within-subjects' factors (two levels each) conducted for the latency, response time, and accommodative response magnitude. The within-subjects' factors were: test \{conventional or hybrid\}, distance $\{$ far or near\}, and direction \{accommodation or disaccommodation\}.

Analogosly, the accommodative dynamics of each possible change in accommodative demand within the hybrid condition was analyzed using a repeated measures ANOVA with two within-subjects' factors: change in accommodative demand and direction. Changes in accommodative demands could occur for one of the following six levels (in increasing order of magnitude): $\{0.17 / 0.50,0.50 / 2.17,0.17 / 2.17,2.17 / 4.50,0.50 /$ $4.50,0.17 / 4.50\}$. This analysis was conducted for the latency, response time, and accommodative response parameters.

Statistical power was assessed with the free open source $\mathrm{G}^{*}$ Power 3.0.10. ${ }^{25}$ Data from a pilot study with 6 subjects were used to compute the required sample size for a statistical power of 0.8. Considering a significance of 0.05 and a paired $t$-test the required sample size was 14 subjects.

\section{Results}

A total of 17 subjects that met the inclusion criteria were tested and included in the analyses. Subjects had a mean age \pm standard deviation of $23 \pm 2$ years, a mean monocular subjective amplitude of accommodation of $11 \pm 3 \mathrm{D}$, and a mean subjective spherical equivalent of $-1.73 \pm 1.68 \mathrm{D}(n=6$ subjects had emmetropia and $n=11$ subjects had myopia). Most of the subjects in our sample had myopia, which is a limitation of the study addressed in the discussion. Myopia was defined as subjective refraction spherical equivalent less than $-0.25 \mathrm{D}$.

\section{Repeatability and agreement between manual flippers and the automated test}

Repeatability of accommodation responses for each condition (1, 2, 3, and 4)

The mean difference \pm standard deviation (SD) between the two sessions (test-retest), the within-subject standard deviation $\left(\mathrm{S}_{\mathrm{W}}\right)$, and the $p$-values obtained with the paired sample $t$-tests are shown in Table 2, described by method and test distance (i.e., conditions 1, 2, 3, and 4).

\section{Agreement of accommodation responses between conditions ( 1 vs. 3 and 2 vs. 4)}

The comparison between the accommodative facility test performed with the manual flipper and the automated method performed with the EOL system is shown in the Bland and Altman plots of Figure 2 for each target distance. As can be appreciated in this figure, the mean difference is increased for near distance for both methods, and subjects were able to complete more $\mathrm{cpm}$ in the automated than in the manual 
Table 2. Repeatability (test-retest) for each method and accommodative distance. diff.: difference. SD: standard deviation. $\mathrm{S}_{\mathrm{W}}$ : within-subject standard deviation. cpm: cycles per minute. * Statistically significant $(p<0.05)$.

\begin{tabular}{|c|c|c|c|c|c|c|}
\hline \multirow[b]{2}{*}{$\begin{array}{l}\text { Test } \\
\text { distance }\end{array}$} & \multicolumn{3}{|c|}{ Manual flippers } & \multicolumn{3}{|c|}{ Automated (EOL system) } \\
\hline & $\begin{array}{c}\text { Mean diff. } \pm \text { SD } \\
{[\text { cpm] }}\end{array}$ & $\begin{array}{c}\mathrm{S}_{\mathrm{W}} \\
{[\mathrm{cpm}]}\end{array}$ & $\begin{array}{c}p- \\
\text { value }\end{array}$ & $\begin{array}{l}\text { Mean diff. } \pm \\
\text { SD [cpm] }\end{array}$ & $\begin{array}{c}\mathrm{S}_{\mathrm{W}} \\
{[\mathrm{cpm}]}\end{array}$ & $\begin{array}{c}p- \\
\text { value }\end{array}$ \\
\hline $\begin{array}{l}\text { Near } \\
\text { Far }\end{array}$ & $\begin{array}{l}-1 \pm 1 \\
-1+1\end{array}$ & $\begin{array}{l}1 \\
1\end{array}$ & $\begin{array}{l}<0.01 * \\
<0.01 *\end{array}$ & $\begin{array}{l}-3 \pm 4 \\
-5+4\end{array}$ & $\begin{array}{l}3 \\
4\end{array}$ & $\begin{array}{l}0.02^{*} \\
<0.01^{*}\end{array}$ \\
\hline
\end{tabular}

flippers tests. Both methods were also statistically compared to paired $t$-tests; $p$-values are also shown in Figure 2.

\section{Hybrid accommodative facility test}

\section{Accommodation response dynamics within condition 5} latency, response time, and accommodative response are shown in Figure 3 and summarized as follows:

For latency of the accommodation/disaccommodation responses, neither the factors (direction and amount of change of the accommodative demand) nor the interaction (direction ${ }^{*}$ change in accommodative demand) resulted in statistically significant differences (Figure 3A).

For the accommodation/disaccommodation response times, a statistically significant $(p<0.05)$ main effect of direction, change in accommodative demand and also the interaction direction ${ }^{*}$ change in accommodative demand was obtained. When controlling for the direction, the Bonferroni post-hoc test showed statistically significant pairwise comparisons when comparing any of the first three levels against any of the remaining three levels for accommodation, and also when comparing the last level against the level four and five for disaccommodation. When controlling for change in accommodative demand, significant pairwise comparisons were obtained in three cases (marked with an asterisk in Figure 3B). The interaction term test ${ }^{\star}$ distance was also significant and the post-hoc test showed significant differences between far and near regardless of the test (conventional or hybrid).

For accommodative response, a statistically significant main effect of direction of accommodation, change in accommodative demand, and also the interaction direction ${ }^{*}$ change in accommodative demand was obtained. In all cases with

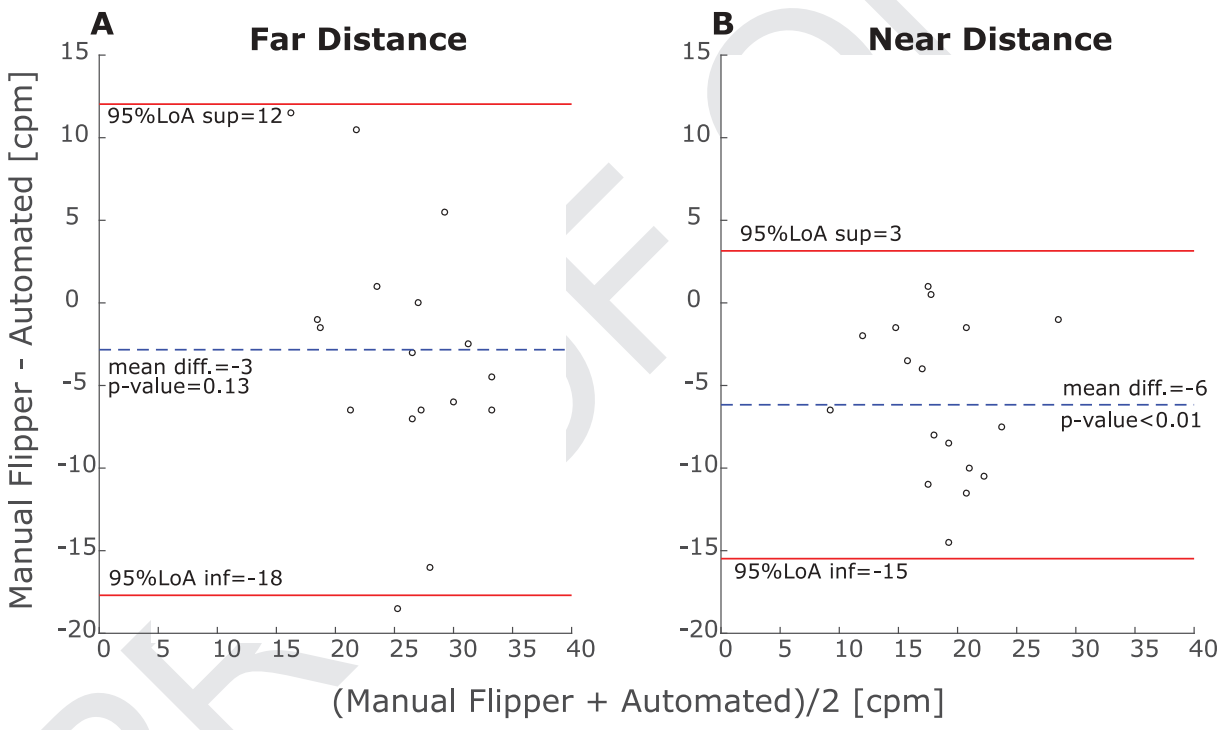

Figure 2. Bland and altman plots with the $95 \%$ limits of agreement (LoA) for far and near distance tests. Sup: superior LoA. Inf: inferior LoA. diff.: difference.
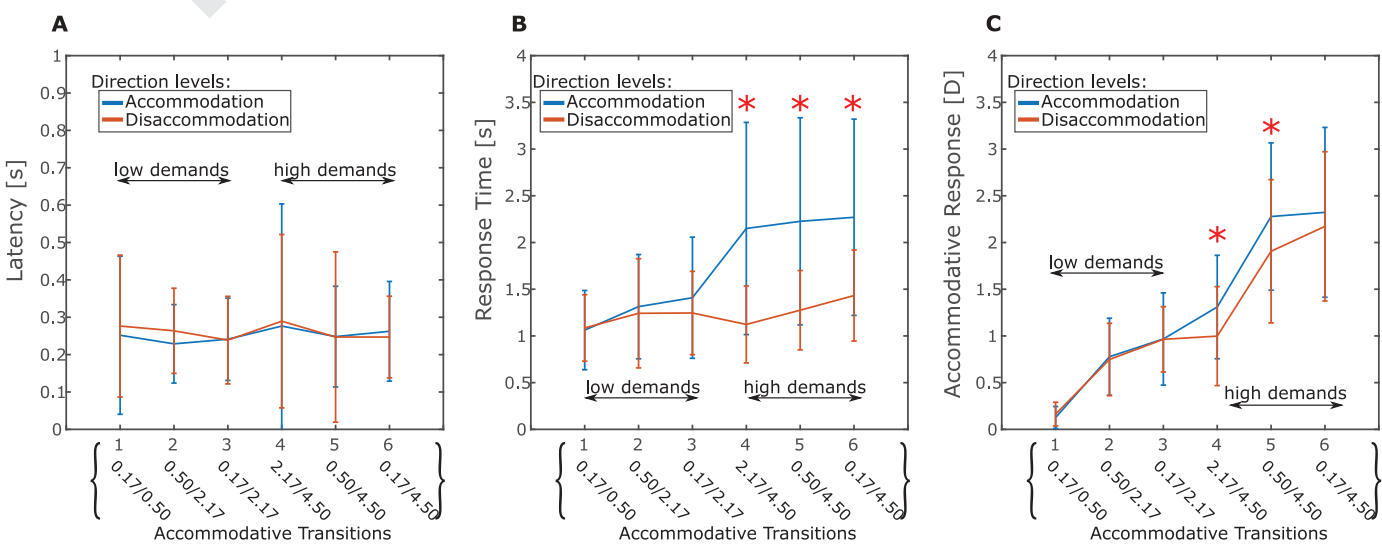

Figure 3. Accommodation dynamics within condition 5. Latency, response time and accommodative response as a function of the accommodative demand factor controlling for direction. Red asterisk indicates statistically significant differences $(p<0.05)$. Error bars are standard deviations. 
$p$-values stronger than 0.01 . When controlling for direction, the Bonferroni post-hoc test showed statistically significant pairwise comparisons in all cases except in the following four cases: (1) between the level two and three for accommodation; (2) between the level five and six for accommodation; (3) between the level two and four for disaccommodation; and (4) between the level three and four for disaccommodation. When controlling for accommodative transition, significant 390 pairwise comparisons were obtained only in two cases that are marked with an asterisk in Figure 3C.

\section{Accommodation dynamics differences among conditions 3, 4 , and 5}

The results of the repeated measures ANOVA with three within-subjects' factors (with two levels each) conducted for the latency, response time, and accommodative response are summarized in Table 3.

Latency is not affected by the predictability of the stimulus, the direction of accommodation, the accommodative demand nor any of the interactions amongst these variables. To the contrary, there is a main effect and interaction of distance and direction in both response time and the accommodative response, the Bonferroni post-hoc tests for the interaction term are shown in Table 4. Additionally, 405 there is a statistically significant difference in the interaction term Test ${ }^{\star}$ Distance for response time. The Bonferroni post-hoc test is shown in Table 5 .

\section{Discussion}

This study compared the repeatability and agreement of clin410 ical manual flippers accommodative facility test with an automated accommodation test performed in a computercontrolled electro-optical system. In addition, a new method for automated accommodative facility tests that presents one of four accommodative demands in an unpredictable manner 415 is presented and analyzed.

Table 3. $P$-values obtained with the repeated measures ANOVA. Statistically significant values are shown in red. *Statistically significant $(p<0.05)$.

\begin{tabular}{lccc}
\hline & Latency & Response time & Accommodative response \\
\hline Test & 0.96 & 0.98 & 0.22 \\
Distance & 0.93 & $<0.01^{*}$ & $<0.01^{*}$ \\
Direction & 0.68 & $0.01^{*}$ & $<0.01^{*}$ \\
Test*Distance & 0.69 & $0.04^{*}$ & 0.49 \\
Test* Direction & 0.36 & 0.21 & 0.91 \\
Distance*Direction & 0.65 & $<0.01^{*}$ & $<0.01^{*}$ \\
Test*Distance* Direction & 0.57 & 0.07 & 0.17 \\
\hline
\end{tabular}

\section{Repeatability and agreement between manual flippers and the automated accommodation facility test}

The agreement level between the manual flipper accommodation facility test and the automated test performed in a computer-controlled electro-optical system is poor for both target distances. The within-subject standard deviation, i.e., repeatability, obtained for both accommodative facility methods is consistent with the value of $3 \mathrm{cpm}$ previously reported ${ }^{26}$ in subjects 8 to 12 years of age. Surprisingly, the automated procedure showed larger inter-subject variability than the manual-flippers procedure. One would expect the inter-subject variability to be smaller in the automated procedure, as the operator's reaction time is not a factor in this procedure. However, the inter-subject variability given by the observer's reaction time appears to surpass that of the operator. There are a number of differences between the manual and automated methods that likely account for the poor agreement found. The most plausible explanation is that the response time of an examiner changing the flipper lenses is much longer, the order of 0.6 seconds/transition, ${ }^{7}$ to that of the automated test (approximately $40 \mathrm{~ms}$ ). Given that young healthy subjects can easily perform 15 to $25 \mathrm{cpm}$ (as shown in Figure 2), the total time spent by the examiner flipping the lenses may add to up to 15 seconds (e.g., $25 \times 0.6=15$ ). Given that the average response time per accommodation change is between 1 and 2.5 seconds (as shown in Figure 3B and previously described), ${ }^{7}$ the number of potential cycles 'gained' in one minute due to automatization would be between 2 to 8 cycles (e.g., $9 /(2 \times 2.5) \approx 2)$. This range accounts for the mean absolute difference in cycles found between the manual and automated (3 and $6 \mathrm{cpm}$ for far and near) accommodative facility tests.

According to these results, accommodative facility measurements obtained from either automatized or manual flippers are not comparable and should not be interchanged. The automated accommodative facility measurements represent more accurate information concerning the individual's ability to accommodate. However, this does not necessarily mean

Table 5. The bonferroni post-hoc test of the significant interaction test*Distance for response time. ${ }^{*}$ Statistically significant $(p<0.05)$.

\begin{tabular}{llcc}
\hline & & \multicolumn{2}{c}{ Response time } \\
\cline { 3 - 4 } & & Mean diff. \pm SD [s] & $p$-value \\
\hline Distance & Test & & \\
$\quad$ Far & Conventional-hybrid & $0.11 \pm 0.27$ & 0.12 \\
$\quad$ Near & Conventional-hybrid & $-0.11 \pm 0.28$ & 0.14 \\
Test & Distance & & \\
$\quad$ Conventional & Far-Near & $-0.21 \pm 0.37$ & $0.03^{*}$ \\
Hybrid & Far-Near & $-0.42 \pm 0.32$ & $<0.01^{*}$ \\
\hline
\end{tabular}

Table 4. The bonferroni post-hoc test of the significant interaction Distance ${ }^{*}$ Direction for response time and the accommodative response. Acc.: Accommodation. Disac.: Disaccommodation. * Statistically significant $(p<0.05)$.

\begin{tabular}{|c|c|c|c|c|c|}
\hline & & \multicolumn{2}{|c|}{ Response time } & \multicolumn{2}{|c|}{ Accommodative response } \\
\hline & & Mean diff. \pm SD [s] & $p$-value & Mean diff. \pm SD $[D]$ & $p$-value \\
\hline Distance & Direction & & & & \\
\hline Far & Acc.-Disacc. & $0.26 \pm 0.77$ & 0.18 & $0.05 \pm 0.19$ & 0.27 \\
\hline Direction & Distance & & & & \\
\hline Accommodation & Far-Near & $-0.56 \pm 0.57$ & $<0.01^{*}$ & $-1.31 \pm 0.38$ & $<0.01^{*}$ \\
\hline Disaccommodation & Far-Near & $-0.07 \pm 0.29$ & 0.33 & $-1.03 \pm 0.51$ & $<0.01^{*}$ \\
\hline
\end{tabular}


that the manual test is inefficient or otherwise inappropriate. Automatization may be best suited for training purposes (e.g., vision therapy or vision training in sports), when subjects have to repeat a specific task several times each day. ${ }^{27}$

\section{The effect of a hybrid, unpredictable, accommodative facility test}

460 In the hybrid approach both far and near accommodative facility tests are automated and integrated into only one test that randomizes among the accommodative demands. One interesting and unexpected outcome was a lack of effect of predictability of the accommodation demand. We initially expected that latency of the 465 accommodation response would be longer for unpredicted than predicted stimuli, but no effect was found. Our initial rationale was originated in a small number of studies carried out more than 40 years ago that concluded a prediction operator in accommodation has a small but considerable impact in latency. ${ }^{8-10}$ However, after a more thorough review of these few manuscripts, it came to light that the results did not warrant the conclusions due to either their very limited sample size (1 to 4 subjects) or the use of nonnaïve subjects (authors were subjects). In addition, the studies are difficult to reproduce due to a lack of specific information about the subjects' characteristics and the instructions they received. Phillips et al. ${ }^{10}$ measured in 1972 the monocular accommodative response in square wave inputs in four subjects and found a mean reduction response latency of $204 \mathrm{~ms}$ when using a predictable square wave stimulus compared to an unpredictable one. As the authors acknowledge in their discussion, the distributions obtained were highly skewed, and the mode difference between the two conditions was minimal, only $49 \mathrm{~ms}$. Two years later, Van der Wildt et al. ${ }^{9}$ investigated the presence of a prediction operator using sinusoid inputs and concluded that even though the effect was small, it was not negligible. They had no explanation as to why subjects did not always succeed in following the stimulus optimally despite its predictability, and noted significant differences in the accommodative response when instructions were changed from "try to fixate the target" to "try to clear the target".

Subsequent studies have shown that the accommodative response and some parameters of its dynamics (e.g., latency) are affected by age, ${ }^{20,28}$ refractive error, ${ }^{7}$ and the task instructions given to participants. ${ }^{29}$ Our hypothesis is that predictability does not affect accommodation responses per se but that specific training using a consistent stimulus and conditions, latency may shorten. Further studies are required to disentangle the isolated effect of stimulus' predictability in time, magnitude, and direction, as well as the interactions of these parameters, on accommodation dynamics.

The second interesting outcome was that accommodation response times and accommodative response levels were affected by the direction of accommodation only for high accommodative demands, not for disaccommodation and not for low accommodation demands. For disaccommodation, the mean response time was around 1 second regardless of the accommodative demand, however, for accommodation, the response time was around 1 second for low accommodative demands and it increased abruptly up to 2.5 seconds for higher demands. Similarly, the differences in accommodation response between accommodation accommodative demand. Despite a large variability across subjects, the differences between accommodation and disaccommodation are statistically significant in both response time and accommodative response magnitude. The results are also consistent with previous studies. ${ }^{7,24}$ Moreover, a linkage between accommodative demand and direction of accommodation also appeared when comparing the hybrid unpredictable test with the automated predictable far and near accommodative facility tests. There was a significant interaction between the test distance and the direction of accommodation in both response time and accommodative response magnitude. Significantly larger responses were obtained for near viewing distances than for far distances during accommodation regardless of the test type (conventional or hybrid). Radhakrishnan et al..$^{7}$ also found significantly longer response times for accommodation than disaccommodation at near distances although this difference was only found in subjects with myopia in their study. Thus, it may be possible that the differences found in our study are also larger due to the number of subjects with myopia in our sample (65\% of the sample). Indeed, the accommodative response is affected not only by experimental conditions, ${ }^{11}$ but also by the observer's refractive error. ${ }^{13}$

In conclusion, our results show that a hybrid unpredictable approach is able to provide a more comprehensive examination of the accommodative capability to change focus over time than the conventional accommodative facility test. Despite the potential advantage of our technique, it is necessary to replicate these results in future studies that include subjects with accommodative dysfunctions and refractive error as covariates. Further work is needed to determine whether the current normative values of accommodative facility should be redefined in the context of the hybrid unpredictable approach, and to determine whether this new test is more sensitive to accommodative anomalies than the conventional clinical accommodative facility tests.

\section{Acknowledgments}

Carles Otero thanks the Generalitat de Catalunya for his awarded Ph.D. studentship.

\section{Funding}

This research was supported by the Spanish Ministry of Economy and Competitiveness under the grant DPI2014-56850-R, the European Union and by Davalor Salud, S.L. None of the institutions had a role in the realization of this manuscript.

\section{Declaration of Interests}

The authors report no conflicts of interest. The authors alone are responsible for the content and writing of the paper.

\section{References}

1. Zellers J, Alpert T, Rouse M. A review of the literature and a normative study of accommodative facility. J Am Optom Assoc. 1984;55:31-37.

2. Scheiman M, Herzberg H, Frantz K, Margolies M. Normative study of accommodative facility in elementary schoolchildren. Am J Optom Physiol Opt. 1988;65:127-34. 
3. Siderov J, DiGuglielmo L. Binocular accommodative facility in prepresbyopic adults and its relation to symptoms. Optom Vis Sci. 1991;68(1):49-53.

4. Thiagarajan P, Ciuffreda KJ. Visual fatigue and accommodative dynamics in asymptomatic individuals. Optom Vis Sci. 2013;90 (1):57-65.

5. Scheiman M, Wick B. Clinical management of binocular vision. 4th ed. Lippincott Williams \& Wilkins; 2014.

6. Sanàbria F, Díaz-Doutón F, Aldaba M, Pujol J. Spherical refractive correction with an electro-optical liquid lens in a double-pass system. J Eur Opt Soc. 2013;8:7-10.

7. Radhakrishnan H, Allen PM, Charman WN. Dynamics of accommodative facility in myopes. Investig Ophthalmol Vis Sci. 2007;48 (9):4375-82.

8. Krishnan V, Phillips S, Stark L. Frequency analysis of accommodation, accommodative vergence and disparity vergence. Vision Res. 1973;13:1545-54.

580 9. Van Der Wildt G, Bouman M, Van De Kraats J. The effect of anticipation on the transfer function of the human lens system. Opt Acta (Lond). 1974;21(11):843-60.

10. Phillips S, Shirachi D, Stark L. Analysis of accommodative response times using histogram information. Am J Optom Arch Am Acad Optom. 1972;49(5):389-401.

11. Otero C, Aldaba M, Vera-Diaz F, Pujol J. Effect of experimental conditions in the accommodation response in myopia. Optom Vis Sci. 2017. doi:10.1097/OPX.0000000000001140.

12. Aldaba M, Otero C, Pujol J, Atchison D. Does the Badal optometer stimulate accommodation accurately? Ophthalmic Physiol Opt. 2017;37(1):88-95.

13. Schmid KL, Strang NC. Differences in the accommodation stimulus response curves of adult myopes and emmetropes: A summary and update. Ophthalmic Physiol Opt. 2015;35(6):613-21.
14. Shibata T, Kim J, Hoffman DM, Banks MS. Visual discomfort with stereo displays: Effects of viewing distance and direction of vergence-accommodation conflict. Proc SPIE Int Soc Opt Eng. 2011;7863:78630P-1-78630P-9.

15. Lambooij M, Ijsselsteijn W, Fortuin M, Heynderickx I. Visual discomfort and visual fatigue of stereoscopic displays: A review. J Imaging Sci Technol. 2009;53(3):30201-30201-14.
16. Schaeffel $\mathrm{F}$, Wilhelm $\mathrm{H}$, Zrenner E. Inter-individual variability in the dynamics of natural accommodation in humans: Relation to age and refractive errors. J Physiol. 1993;32:301-20.

17. Aldaba M, Gómez-López S, Vilaseca M, Pujol J, Arjona M. Comparing autorefractors for measurement of accommodation. Optom Vis Sci. 2015;92(10):1003-11.

18. Otero C, Aldaba M, Martínez-Navarro B, Pujol J. Effect of apparent depth cues on accommodation in a Badal optometer. Clin Exp Optom. 2017. doi:10.1111/cxo.12534.

19. Charman WN, Tucker J. Dependence of the accommodation response on the spatial frequency spectrum of the observed object. Vision Res. 1977;17(1):129-39.

20. Kasthurirangan S, Glasser A. Age related changes in accommodative dynamics in humans. Vision Res. 2006;46(8-9):1507-19.

21. Bharadwaj SR, Schor CM. Acceleration characteristics of human ocular accommodation. Vision Res. 2005;45(1):17-28.

22. Bernal-Molina P, Marín-Franch I, Del Aguila-Carrasco AJ, et al. Human eyes do not need monochromatic aberrations for dynamic accommodation. Ophthalmic Physiol Opt. 2017:1-8. doi:10.1111/ opo. 12398 .

23. Momeni-Moghaddam H, Kundart J, Askarizadeh F. Comparing measurement techniques of accommodative amplitudes. Indian J Ophthalmol. 2014;62:683-87.

24. Kasthurirangan S, Vilupuru AS, Glasser A. Amplitude dependent accommodative dynamics in humans. Vision Res. 2003;43 (27):2945-56.

25. Faul F, Erdfelder E, Lang A-G, Buchner A. G*Power 3: A flexible statistical power analysis program for the social, behavioral, and biomedical sciences. Behav Res Methods. 2007;39(2):175-91.

26. McKenzie K, Kerr S, Rouse M, DeLand P. Study of accommodative facility testing reliability. Am J Optom Physiol Opt. 1987;64(3):186-94.

27. Ciuffreda K, Wang B. Vision training and sports. In: Hung G, Pallis J, editors. Biomedical engineering principles in sports. 1st ed. Boston, MA: Springer; 2004.

28. Mordi JA, Ciuffreda KJ. Dynamic aspects of accommodation: Age and presbyopia. Vision Res. 2004;44(6):591-601.

29. Stark L, Atchison D. Subject instructions and methods of target presentation in accommodation research. Invest Ophthalmol Vis Sci. 1994;35(2):528-37. 